\title{
A Novel Ring Shaped Photodiode for Reflectance Pulse Oximetry in Wireless Applications
}

Duun, Sune; Haahr, Rasmus Grønbek; Birkelund, Karen; Raahauge, P.; Petersen, P.; Dam, H; Noergaard, L.; Thomsen, Erik Vilain

Published in:

Proceeedings of the 6th IEEE Conference on Sensors

Link to article, DOI:

10.1109/ICSENS.2007.4388469

Publication date:

2007

Document Version

Publisher's PDF, also known as Version of record

Link back to DTU Orbit

Citation (APA):

Duun, S., Haahr, R. G., Birkelund, K., Raahauge, P., Petersen, P., Dam, H., Noergaard, L., \& Thomsen, E. V. (2007). A Novel Ring Shaped Photodiode for Reflectance Pulse Oximetry in Wireless Applications. In Proceeedings of the 6th IEEE Conference on Sensors (pp. 596-599). IEEE.

https://doi.org/10.1109/ICSENS.2007.4388469

\section{General rights}

Copyright and moral rights for the publications made accessible in the public portal are retained by the authors and/or other copyright owners and it is a condition of accessing publications that users recognise and abide by the legal requirements associated with these rights.

- Users may download and print one copy of any publication from the public portal for the purpose of private study or research.

- You may not further distribute the material or use it for any profit-making activity or commercial gain

- You may freely distribute the URL identifying the publication in the public portal 


\title{
A Novel Ring Shaped Photodiode for Reflectance Pulse Oximetry in Wireless Applications
}

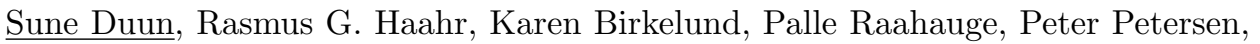 \\ Henrik Dam, Lars Noergaard, and Erik V. Thomsen
}

\begin{abstract}
We present a pulse oximeter for use in homecare applications in a sticking patch with integrated electronics. The core in the pulse oximeter is a large ring shaped backside silicon pn photodiode placed around a Ledtronics dual LED with wavelengths of $660 \mathrm{~nm}$ and 940 nm. The concentric photodiode gives optimal gathering of light and thereby enabling lower LED drive currents and lower power consumption. To further optimize the photodiode a two layer $\mathrm{SiO}_{2} / \mathrm{SiN}$ interference filter is employed yielding $98 \%$ transmission at the wavelengths of the LED and damping of other wavelengths. The presented photodiode has an inner-outer radius of $3.29-4.07 \mathrm{~mm}$ and an area of $18 \mathrm{~mm}^{2}$; however, photodiodes with ring center radii ranging from $2.8-4.9 \mathrm{~mm}$ have been fabricated. Using the pulse oxymetry sensor photoplethysmograms clearly showing the cardiovascular cycle are recorded. An on-chip integrated $\mathrm{Au}$ thermistor for surface body temperature measurements is found to have a repeatability of $\pm 0.26{ }^{\circ} \mathrm{C}$.
\end{abstract}

\section{INTRODUCTION}

Continuously health monitoring of hospitalized persons and people working in high risk environments has recently gained interest due to the availability of miniaturized sensors for construction of small and autonomous sensor systems. We have previously presented an idea for a small system for this purpose - the Electronic Patch [1], Fig. 1. The system contains a reflective pulse oximeter based on a custom designed photodiode and a dual LED. It will be integrated into a sticking patch with an ASIC for signal processing and wireless communication based on a SWM1601 radio chip. The electronic patch is powered by an CR-2025 coin battery and is intended for use on chronic lung disease patients hospitalized in their own home and firefighters.

In the literature reflection mode pulse oximetry sensors have previously been reported: Studies have been conducted using one photo detector and one light source by Mendelson et al. [2] and Dassel et al. [3], an early design using multiple photo detectors with a single light source was made by Mendelson et al. [4] and more recently power optimization has been studied [5]. Some other design approaches have also been studied: Takatani et al. have studied a design using 20 LEDs placed concentrically around a single photo detector [6], [7] and a finger-ring type of pulse oximeter have been developed and studied by both Asada et al. [8], [9] and Mendelson et al. [10].

We report a pulse oximeter based on a novel ring-shaped photodiode with a hole in the middle for the light source

This work was supported by the Danish Ministry of Science, Technology and Innovation.

The authors are all with the Department of Micro and Nanotechnology, Technical University of Demark, 2800 Kgs. Lyngby, Denmark. sdu@mic.dtu.dk

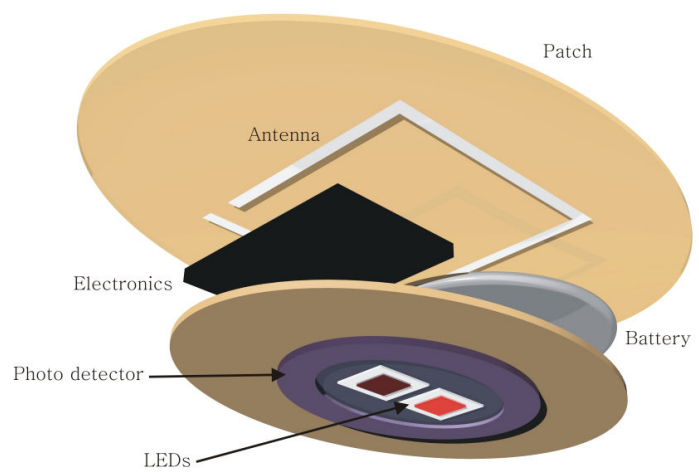

Fig. 1. Illustration of the developed electronic patch. The patch has a size of $20 \mathrm{~cm}^{2}$ and is $5 \mathrm{~mm}$ thick.

as show on Fig. 1. The photodiode collects backscattered light from the tissue all the way around the light source, therefore as much as possible of the backscattered light will be collected by the photodiode. This allows for a very low LED drive current which will lower the power consumption by the device. Furthermore we have integrated an anti-reflection optical filter on the diode for enhanced sensitivity. In addition to the photodiode the chip also contains a thermistor for measuring the body surface temperature, thereby creating a multi sensor for medical purposes on a single chip. The Electronic Patch packaging solution allows discreet placement under the clothes and low power usage.

\section{A. Pulse Oximetry}

Pulse oximetry is a spectrophotometric method for noninvasive measurement of the arterial oxygen saturation, $\mathrm{SpO}_{2}$, and pulse. It can generally be performed in either transmission or reflection modes. In the first light is transmitted through the tissue e.g. a finger and in the latter light is backscattered from the tissue. The method relies on difference in the absorption spectra of oxygenated and de-oxygenated hemoglobin. The ratio between these are seen on Fig. 2. It is seen that the ratio has a peak at approximately $660 \mathrm{~nm}$ and at higher wavelengths the ratio is lower than one. Conventionally two wavelengths at $660 \mathrm{~nm}$ (RED) and $940 \mathrm{~nm}$ (IR) are used since the absorption ratio is large and small at those wavelengths respectively. This minimizes the uncertainty of the $\mathrm{SpO}_{2}$ measurement.

A photoplethysmogram is an optical recording of the cardiovascular cycle. The $\mathrm{SpO}_{2}$ is a function of the measured magnitude at the systolic and diastolic states on the two 


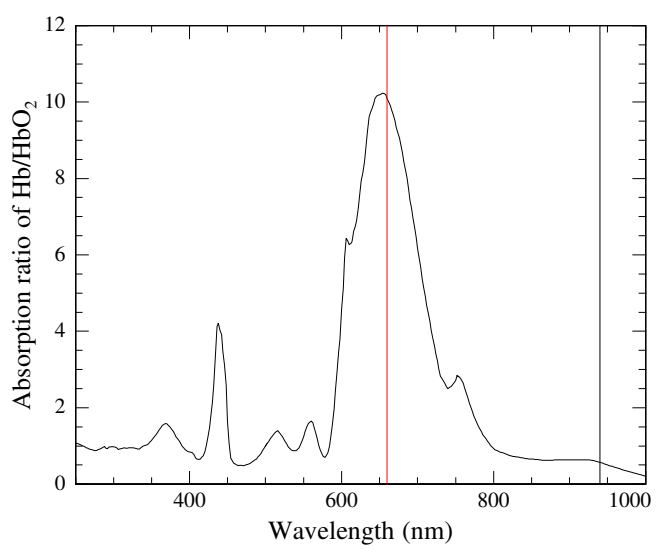

Fig. 2. Ratio between the absorption coefficients of de-oxygenated and oxygenated hemoglobin. The plot is based on data from [11].

photoplethysmograms (RED and IR):

$$
\mathrm{SpO}_{2} \sim \frac{\ln \left(\frac{\mathrm{RED}_{\text {systole }}}{\mathrm{RED}_{\text {diastole }}}\right)}{\ln \left(\frac{\mathrm{IR} \text { diastole }}{I \mathrm{R}_{\text {diastole }}}\right)}
$$

where $\mathrm{RED}_{\text {systole }}$ and $\mathrm{RED}_{\text {diastole }}$ are the magnitudes of the red light measured at the systolic and diastolic states respectively and likewise for $\mathrm{IR}_{\text {systole }}$ and $\mathrm{IR}_{\text {diastole }}$. However, the relation has to be empirically calibrated for the specific device [12].

\section{DESIGN OF CHIP AND PHOTODIODE}

The multi sensor chip with the photodiode and thermistor developed in this work have several novel features. The frontand backside of the chip together with a Ledtronics dual LED with wavelengths of $660 \mathrm{~nm}$ and $940 \mathrm{~nm}$ is seen on Fig. 3 . The chip has a hole in the middle of $3.3 \times 3.6 \mathrm{~mm}^{2}$ which fits to the dual LED. The photodiode is defined as a single ring-shaped structure concentrically around the hole with an active area of $18 \mathrm{~mm}^{2}$. The $\mathrm{p}-\mathrm{n}$ diode junction is made on the contact side of the chip facing away from the skin. Using a backside photodiode makes it possible to avoid metallization and wiring to the surface, filter side, facing the skin. In this backside photodiode photons are absorbed by generation of minority carriers at the front side, filter side, which then diffuse to the diode junction on the backside, contact side. Therefore, the diode is fabricated in p-type Silicon since minority electrons have approximately a factor of 2 higher diffusivity compared to holes. Also a substrate with a high minority carrier lifetime must be used.

The front side of the chip has a anti-reflection filter which allows transmission of $98 \%$ for wavelengths of $660 \mathrm{~nm}$ and 940 $\mathrm{nm}$ which enhance the sensitivity of the device, Fig. 4. The filter is designed so that other wavelengths are suppressed to screen off stray light. The peak at $515 \mathrm{~nm}$ is less important due to a shorter penetration photon penetration depth in the base material and a high absorbance by tissue at shorter wavelengths [11]. The filter is made by a stack of $50 \mathrm{~nm}$ silicon dioxide and $550 \mathrm{~nm}$ silicon nitride. The first $800 \mathrm{~nm}$ silicon below the filter is highly boron doped to reduce the minority carrier electron concentration at the surface and thereby also reducing the surface recombination.

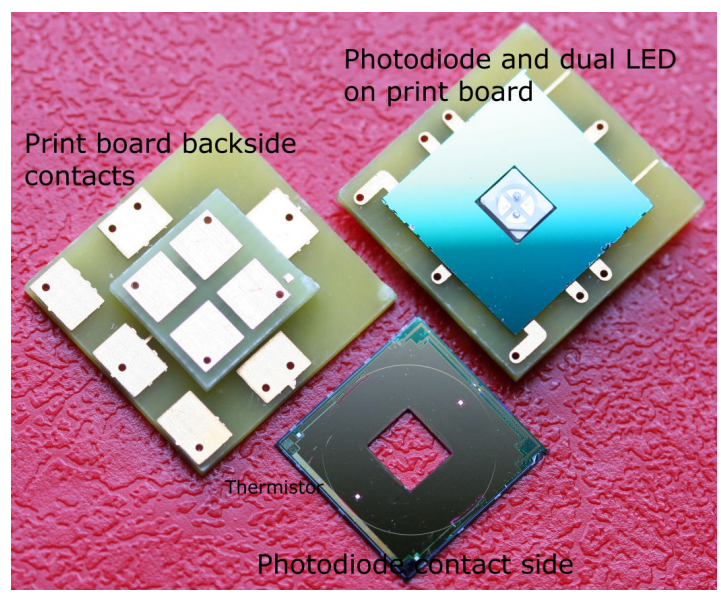

Fig. 3. Picture of photodiode chip with the ring-shape photodiode mounted on a small print board together with the dual LED. The chip is flip chip mounted with the filter side facing up. Contacts for both photodiode and LED is on the back side of the print board.

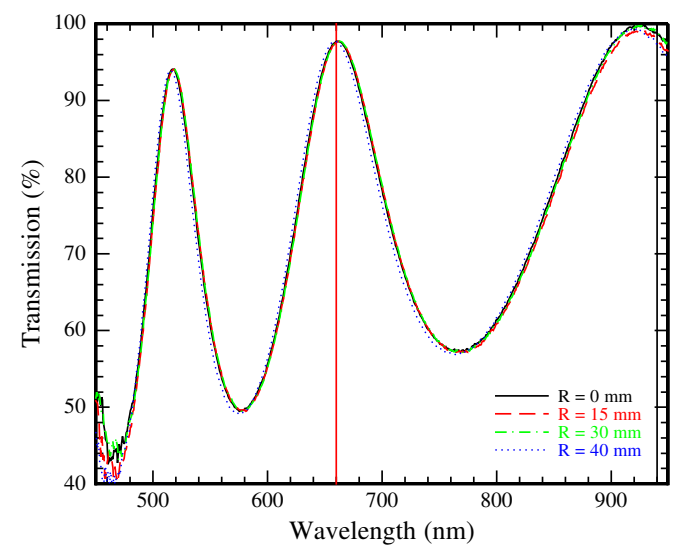

Fig. 4. Transmission of the $\mathrm{SiO}_{2}$ and $\mathrm{SiN}$ interference filter measured with a Filmtek reflectometer. The transmission uniformity across a wafer is seen to be good since there is now significant deviation in transmission between the four plots measured at a radius, $R$, from the wafer center.

The chip has an Au thermistor integrated, it is made in a meander structure with a total length of $17.8 \mathrm{~cm}$, a width of $3 \mu \mathrm{m}$ and an $\mathrm{Au}$ thickness of $200 \mathrm{~nm}$ plus $20 \mathrm{~nm} \mathrm{Cr}$ for adhesion.

\section{FABRICATION}

The starting point is a $300 \mu \mathrm{m}$ thick 4" p-type Si floatzone wafer with $1 \mathrm{~ms}$ minority carrier lifetime. A $360 \mathrm{~nm}$ thermal wet silicon oxide is grown and alignment marks and the photodiode areas are defined using photolithography and the oxide is etched in a STS Reactive Ion Etcher (RIE). A $1 \mathrm{~h}$ phosphor pre-deposition is done at $1000{ }^{\circ} \mathrm{C}$, Fig. 5(a).

Another $1 \mathrm{~h}$ wet oxidation at $1000{ }^{\circ} \mathrm{C}$ is performed to transfer alignment marks and make a masking silicon oxide for boron pre-deposition of contacts. The contacts are defined using photolithography and holes in the masking silicon oxide are opened with bHF. The boron pre-deposition is performed 
for $1 \mathrm{~h}$ at $975{ }^{\circ} \mathrm{C}$, Fig. $5(\mathrm{a})$, giving a $1 \mu \mathrm{m}$ deep contact doping. In this step the filter side doping is also performed.

The masking silicon oxide is removed in bHF and a $50 \mathrm{~nm}$ dry silicon oxide is grown on both sides of the wafer. One the skin side it serves as the first filter layer and to give a good surface passivation giving a low surface recombination velocity and on the diode side it serves as electrical isolation. As high temperature processes are performed it is extremely important to avoid contamination since this will degrade the diode characteristic and quantum efficiency. Therefore, only clean machines, baths and tools are used.

On top of the silicon oxide a $550 \mathrm{~nm}$ PECVD silicon nitride is deposited for the second filter layer and electric passivation, Fig. 5(b). The filter transmission curve is controlled on a Filmtek reflectometer and is shown in Fig. 4.

Contact holes are defined using photolithography and opened with RIE. Thereafter, the Au thermistor, and metallization are defined using photolithography and $20 \mathrm{~nm} \mathrm{Cr}$ and $200 \mathrm{~nm} \mathrm{Au}$ are e-beam evaporated on the wafer using an Alcatel e-beam evaporator, Fig. 5(c). The Chromium is used to give a good adhesion.

The hole for the dual LED is defined using backside photolithography and a hole is etched through the wafer from the skin side with a STS Advanced Silicon Etcher, ASE, Fig. 5(d).

The diodes are annealed at $250{ }^{\circ} \mathrm{C}$ in nitrogen ambient to ensure low contact resistance. Finally they are diced out. The location of doped areas and contact on the contact side are shown in Fig. 5(e), here the isolation $\mathrm{SiO}_{2}$ is not shown.

\section{Results}

\section{A. Diode characteristics}

A current voltage characteristics for the photodiode is shown in Fig. 6. The photodiode has a forward saturation current density of $\mathrm{J}_{\mathrm{s}}=167 \mathrm{pA} / \mathrm{cm}^{2}$ and an ideality factor of $\mathrm{n}=1.19$, showing that the diode is close to ideal. Some generation current is observed as seen from the deviation from the fit in the range 0 to $0.12 \mathrm{~V}$. The series resistance of the mounted chip on a printed circuit board is $R_{\mathrm{S}}=84 \Omega$.

Quantum efficiencies of $67 \%$ and $70 \%$ have been obtained at wavelengths of $660 \mathrm{~nm}$ and $940 \mathrm{~nm}$ respectively without the two layer interference filter but with a single $140 \mathrm{~nm}$ thick $\mathrm{SiO}_{2}$ layer for anti reflection. This can be seen in Fig. 7 where the transmission characteristic of the filter is also shown. The quantum efficiency is reasonable for the current design, but somewhat less than that of commercial photodiodes.

\section{B. Au thermistor characteristics}

The thermistor is found to have a resistance of $13.6 \mathrm{k} \Omega$ at $37^{\circ} \mathrm{C}$ and a TCR of $2.7 \cdot 10^{-3} \mathrm{~K}^{-1}$, which is a little less than that of pure $\mathrm{Au}\left(3.4 \cdot 10^{-3} \mathrm{~K}^{-1}\right)$ and it is believed to be due to surface effects and a non lattice structure. The repeatability of temperature measurements with the thermistor was found to be $\pm 0.26{ }^{\circ} \mathrm{C}$.

\section{Photoplethysmograms}

The fabricated photodiode packaged with a dual LED, as shown on Fig. 3, has been tested as a pulse oximeter on a test person's left hand index finger at the opposite site of the nail. The result is shown in Fig. 8. The two recorded photoplethysmograms for the RED and IR light both clearly

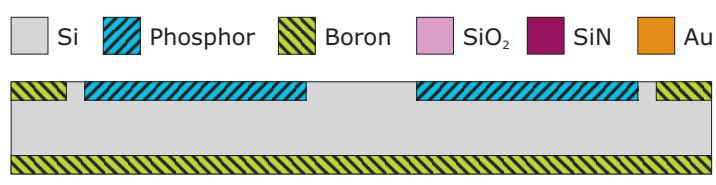

(a) Phosphor and boron doping of contacts.

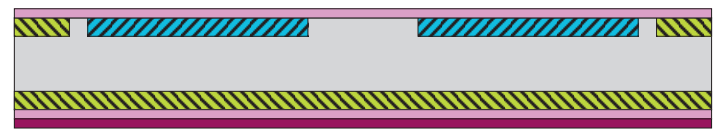

(b) $\mathrm{SiO}_{2} / \mathrm{SiN}$ optical filter.

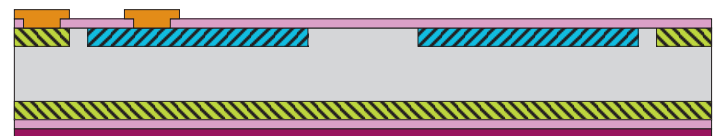

(c) Contact and thermistor metallization.

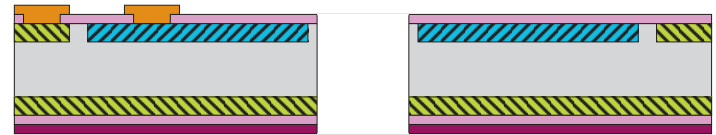

(d) ASE etch of hole for LED's.

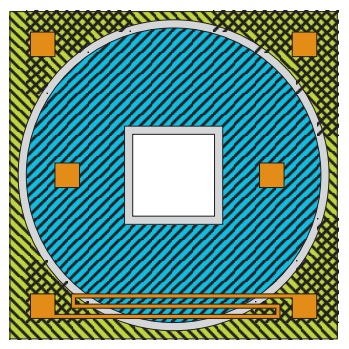

(e) The contact side. Insulating $\mathrm{SiO}_{2}$ is not shown.

Fig. 5. Process sequence for ring shaped photodiode for pulseoximetry.

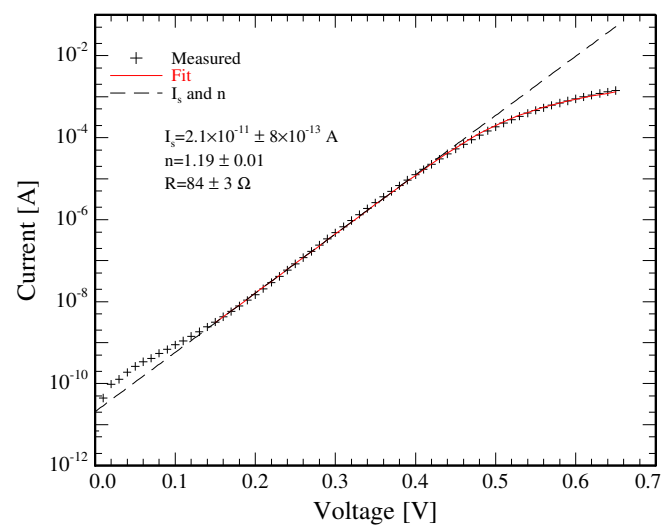

Fig. 6. Current-voltage characteristic of the fabricated photodiode. The photodiode ring has inner radius $3.29 \mathrm{~mm}$ and outer radius 4.07 $\mathrm{mm}$ yielding an active area of $18 \mathrm{~mm}^{2}$. 


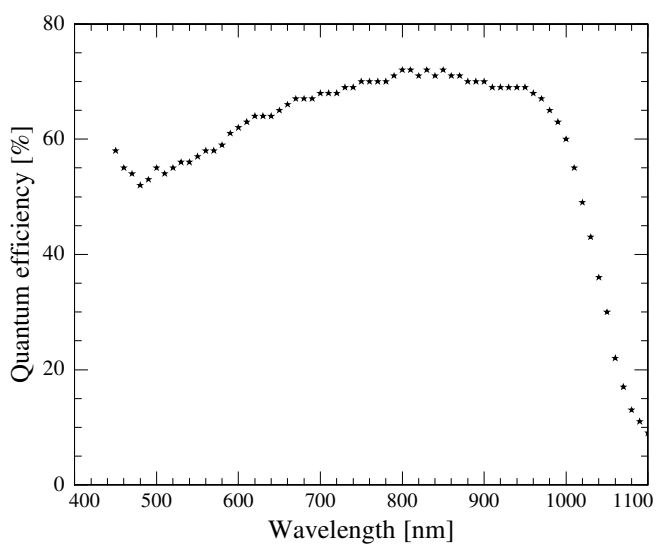

Fig. 7. Quantum efficiency of photodiode with a single $140 \mathrm{~nm}$ $\mathrm{SiO}_{2}$ layer interference filter.

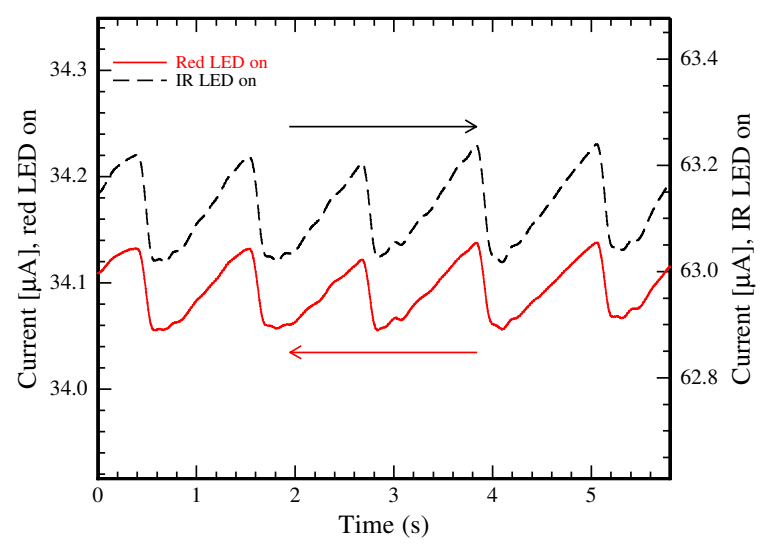

Fig. 8. Photoplethysmograms measured with the developed ringshaped photodiode.

display the cardiovascular cycle. The data are obtained by blinking with the red and IR LED elements with a frequency of $4 \mathrm{kHz}$ and $180^{\circ}$ out of phase. Data are sampled at $8 \mathrm{kHz}$. The high sampling frequency ensures a detailed measurement of the cardiovascular cycle. The data collection is done using Labview and the data have been processed with a moving average using a rectangular window with a width of 401 data points. The curve is also corrected for drift.

The resulting photodiode current for the red light is shown on the left of the figure and the IR on the right. Using (1) a ratio of 0.7 is obtained. In [12] a simple theoretical model for the calibration is derived. Using this it is found that the ratio in Eqn. 1 of 0.7 corresponds to a $\mathrm{SpO}_{2}$ value of approximately $85 \%$. Even though the pulse oximeter has not been empirically calibrated in a clinical setup the result indicates that it works properly.

\section{CONClusion And Future WORKS}

\section{A. Conclusions}

A multisensor chip for measurements of temperature and pulse oximetry $\left(\mathrm{SpO}_{2}\right)$ is reported. The chip has a novel ringshaped backside photodiode with a special designed optical filter for pulse oximetry in low-power applications, and an $\mathrm{Au}$ thermistor. The design, fabrication and charaterization of the photodiode and thermistor are presented and it is proved to work as a pulse oximeter. The chip has been packaged together with a dual LED of $660 \mathrm{~nm}$ and $940 \mathrm{~nm}$ into a compact pulse oximeter which is compatible with integration into the electronic patch, Fig. 1.

The measuring site on the body is an important parameter for the pulse oximetry [5]. We have not yet studied measuring sites with the developed pulse oximeter for the electronic patch and more studies should be done in this direction to optimize the electronic patch solution. However, the ringshaped photodiode has been fabricated with various radii $(2.8$ $\mathrm{mm}-4.9 \mathrm{~mm}$ ) and studies of optimal detector sizes at different measuring sites can therefore been conducted.

\section{B. Future Works}

The pulse oximeter should be completely integrated into the electronic patch for optimizing the systems with respect to measuring site, photodiode geometry and power consumption. The monitoring systems should then be evaluated in clinical trials.

\section{ACKNOWLEDGMENTS}

The authors gratefully acknowledge the The Ministry of Science, Technology and Innovation for financing this research project.

\section{REFERENCES}

[1] E. V. Thomsen, A. Hyldgård, K. Birkelund, R. G. Haahr, S. Duun, R. Elsubaihi, J. Branebjerg, "Distributed sensors: Applications, fabrication and challenges", Proc. Eurosensors $X X, 2006$

[2] Y. Mendelson, "Noninvasive pulse oximetry utilizing skin reflectance photoplethysmography", IEEE Transactions on Biomedical Engineering, vol. 35, nr. 10, pp 798-805, 1988

[3] A.C. Dassel, "Reflectance Pulse oximetry in fetal lambs", $P e-$ diatr. Res., vol. 31, nr. 6 pp 266-269, 1992

[4] Y. Mendelson, "Design and evaluation of a new reflectance pulse oximeter sensor", Medical Instrumentation, vol. 22, nr. 4, pp 167-173, 1988

[5] Y. Mendelson, "Measurement site and photodetector size considerations in optimizing power consuption of a wearable reflectance pulse oximeter", Proc. 25th Annual IEEE EMBS conf., pp 3016-3019, 2003

[6] S. Takatani, "A new hybrid reflectance optical pulse oximetry sensor for lower oxygen saturation measurements and for broader clinical applications", Proc. SPIE, nr. 2976, pp 78-87, 1997

[7] S. Takatani, "A novel hybrid reflectance pulse oximeter sensor with improved linearity and general applicability to various portions of the body", Proc. 20th Annual IEEE EMBS conf., vol. 20, nr. 4, pp 1858-1861, 1998

[8] H. Asada, "The Ring Sensor: a New Ambulatory Wearable Sensor for Twenty-Four Hour Patient Monitoring", Proc. 20th Annual IEEE EMBS conf., vol. 4, pp 1906-1909, 1998

[9] H. Asada, "Artifact-resistant power-efficient design of fingerring plethysmographic sensors", IEEE Transactions on Biomedical Engineering, vol. 48, nr. 7, pp 795-805, 2001

[10] Y. Mendelson, "Minimazation of LED power consumption in the design of a wearable pulse oximeter", Proc. IASTED Conf. on Biomedical Engineering, pp 249-254, 2003

[11] Scott Prahl, Oregon Medical Laser Center, http://omlc.ogi.edu, Portland, OR, 1998.

[12] J. G. Webster, Design of Pulse Oximeters, IOP Publishing, Bristol, UK; 1997. 